\title{
On the solutions of some nonlinear systems of difference equations
}

\author{
Elsayed M Elsayed ${ }^{1,3^{*}}$ and Hamdy El-Metwally ${ }^{2,3}$
}

\begin{tabular}{l}
\hline "Correspondence: \\
emelsayed@mans.edu.eg; \\
emmelsayed@yahoo.com \\
${ }^{1}$ King Abdulaziz University, Faculty \\
of Science, Mathematics \\
Department, P.O. Box 80203, Jeddah \\
21589, Saudi Arabia \\
${ }^{3}$ Department of Mathematics, \\
Faculty of Science, Mansoura \\
University, Mansoura 35516, Egypt \\
Full list of author information is \\
available at the end of the article
\end{tabular}

\begin{abstract}
In this paper, we deal with the existence of solutions and the periodicity character of the following systems of rational difference equations with order three:

$$
x_{n+1}=\frac{x_{n} y_{n-2}}{y_{n-1}\left( \pm 1 \pm x_{n} y_{n-2}\right)}, \quad y_{n+1}=\frac{y_{n} x_{n-2}}{x_{n-1}\left( \pm 1 \pm y_{n} x_{n-2}\right)}
$$

where the initial conditions $x_{-2}, x_{-1}, x_{0}, y_{-2}, y_{-1}$ and $y_{0}$ are nonzero real numbers.

MSC: $39 \mathrm{~A} 10$

Keywords: difference equations; periodic solution; solution of difference equation; system of difference equations
\end{abstract}

\section{Introduction}

Recently, rational difference equations have attracted the attention of many researchers for various reasons. On the one hand, they provide examples of nonlinear equations which are, in some cases, treatable but their dynamics present some new features with respect to the linear case. On the other hand, rational equations frequently appear in some biological models. Hence, their study is of interest also due to their applications. A good example of both facts is Ricatti difference equations because the richness of the dynamics of Ricatti equations is very well known (see, e.g., [1,2]), and a particular case of these equations provides the classical Beverton-Holt model on the dynamics of exploited fish populations [3]. Obviously, higher-order rational difference equations and systems of rational equations have also been widely studied but still have many aspects to be investigated. The reader can find in the following books [4-6], and works cited therein, many results, applications, and open problems on higher-order equations and rational systems.

A preliminary study of planar rational systems can be found in the paper [7] by Camouzis et al. In the work, they give some results and provide some open questions for systems of equations of the following type:

$$
x_{n+1}=\frac{\alpha_{1}+\beta_{1} x_{n}+\gamma_{1} y_{n}}{A_{1}+B_{1} x_{n}+C_{1} y_{n}}, \quad y_{n+1}=\frac{\alpha_{2}+\beta_{2} x_{n}+\gamma_{2} y_{n}}{A_{2}+B_{2} x_{n}+C_{2} y_{n}}, \quad n=0,1,2, \ldots,
$$

where the parameters are taken to be nonnegative. As shown in the cited paper, some of these systems can be reduced to some Ricatti equations or to some previously studied second-order rational equations. Furthermore, for some choices of the parameters, one

C 2013 Elsayed and El-Metwally; licensee Springer. This is an Open Access article distributed under the terms of the Creative Commons Attribution License (http://creativecommons.org/licenses/by/2.0), which permits unrestricted use, distribution, and reproduction in any medium, provided the original work is properly cited. 
obtains a system which is equivalent to the case with some other parameters. Camouzis et $a l$. arrived at a list of 325 non-equivalent systems that should be focused on. They list such systems as pairs $k, l$, where $k$ and $l$ make reference to the number of the corresponding equation in their Tables 3 and 4 . There are many papers that are related to the difference equations systems. For example, the periodicity of the positive solutions of the rational difference equations systems

$$
x_{n+1}=\frac{m}{y_{n}}, \quad y_{n+1}=\frac{p y_{n}}{x_{n-1} y_{n-1}}
$$

has been obtained by Cinar in [8].

In [9] Clark and Kulenovic investigated the global asymptotic stability of the system

$$
x_{n+1}=\frac{x_{n}}{a+c y_{n}}, \quad y_{n+1}=\frac{y_{n}}{b+d x_{n}} .
$$

The behavior of the positive solutions of the following system:

$$
x_{n+1}=\frac{x_{n-1}}{1+x_{n-1} y_{n}}, \quad y_{n+1}=\frac{y_{n-1}}{1+y_{n-1} x_{n}}
$$

has been studied by Kurbanli et al. [10].

Touafek et al. [11] investigated the periodic nature and got the form of the solutions of the following systems of rational difference equations:

$$
x_{n+1}=\frac{x_{n-3}}{ \pm 1 \pm x_{n-3} y_{n-1}}, \quad y_{n+1}=\frac{y_{n-3}}{ \pm 1 \pm y_{n-3} x_{n-1}} \text {. }
$$

In [12] Yalçınkaya investigated the sufficient conditions for the global asymptotic stability of the following system of difference equations:

$$
x_{n+1}=\frac{x_{n}+y_{n-1}}{x_{n} y_{n-1}-1}, \quad y_{n+1}=\frac{y_{n}+x_{n-1}}{y_{n} x_{n-1}-1} \text {. }
$$

Similarly, difference equations and nonlinear systems of the rational difference equations were investigated, see [1-39].

In this paper, we investigate the periodic nature and the form of the solutions of some nonlinear difference equations systems of order three

$$
x_{n+1}=\frac{x_{n} y_{n-2}}{y_{n-1}\left( \pm 1 \pm x_{n} y_{n-2}\right)}, \quad y_{n+1}=\frac{y_{n} x_{n-2}}{x_{n-1}\left( \pm 1 \pm y_{n} x_{n-2}\right)},
$$

where the initial conditions $x_{-2}, x_{-1}, x_{0}, y_{-2}, y_{-1}$ and $y_{0}$ are nonzero real numbers.

Definition (Periodicity) A sequence $\left\{x_{n}\right\}_{n=-k}^{\infty}$ is said to be periodic with period $p$ if $x_{n+p}=$ $x_{n}$ for all $n \geq-k$.

2 On the solution of the system $x_{n+1}=\frac{x_{n} y_{n-2}}{y_{n-1}\left(1+x_{n} y_{n-2}\right)}, y_{n+1}=\frac{y_{n} x_{n-2}}{x_{n-1}\left(1+y_{n} x_{n-2}\right)}$ In this section, we investigate the solutions of the two difference equations system

$$
x_{n+1}=\frac{x_{n} y_{n-2}}{y_{n-1}\left(1+x_{n} y_{n-2}\right)}, \quad y_{n+1}=\frac{y_{n} x_{n-2}}{x_{n-1}\left(1+y_{n} x_{n-2}\right)},
$$

where $n \in \mathbb{N}_{0}$ and the initial conditions are arbitrary nonzero real numbers. 
Theorem 1 Assume that $\left\{x_{n}, y_{n}\right\}$ are solutions of system (1). Then for $n=0,1,2, \ldots$, we see that all solutions of system (1) are given by the following formulas:

$$
\begin{aligned}
& x_{4 n-2}=\frac{a^{n} f^{n}}{c^{n-1} d^{n}} \prod_{i=0}^{n-1} \frac{(1+(4 i) c d)}{(1+(4 i+2) a f)}, \quad x_{4 n-1}=\frac{b a^{n} f^{n}}{c^{n} d^{n}} \prod_{i=0}^{n-1} \frac{(1+(4 i+1) c d)}{(1+(4 i+3) a f)}, \\
& x_{4 n}=\frac{a^{n+1} f^{n}}{c^{n} d^{n}} \prod_{i=0}^{n-1} \frac{(1+(4 i+2) c d)}{(1+(4 i+4) a f)}, \quad x_{4 n+1}=\frac{a^{n+1} f^{n+1}}{e c^{n} d^{n}(1+a f)} \prod_{i=0}^{n-1} \frac{(1+(4 i+3) c d)}{(1+(4 i+5) a f)}
\end{aligned}
$$

and

$$
\begin{aligned}
& y_{4 n-2}=\frac{c^{n} d^{n}}{a^{n} f^{n-1}} \prod_{i=0}^{n-1} \frac{(1+(4 i) a f)}{(1+(4 i+2) c d)}, \quad y_{4 n-1}=\frac{e c^{n} d^{n}}{a^{n} f^{n}} \prod_{i=0}^{n-1} \frac{(1+(4 i+1) a f)}{(1+(4 i+3) c d)}, \\
& y_{4 n}=\frac{c^{n} d^{n+1}}{a^{n} f^{n}} \prod_{i=0}^{n-1} \frac{(1+(4 i+2) a f)}{(1+(4 i+4) c d)}, \quad y_{4 n+1}=\frac{c^{n+1} d^{n+1}}{b a^{n} f^{n}(1+c d)} \prod_{i=0}^{n-1} \frac{(1+(4 i+3) a f)}{(1+(4 i+5) c d)}
\end{aligned}
$$

where $x_{-2}=c, x_{-1}=b, x_{0}=a, y_{-2}=f, y_{-1}=e$ and $y_{0}=d$.

Proof For $n=0$ the result holds. Now suppose that $n>0$ and that our assumption holds for $n-1$, that is,

$$
\begin{aligned}
& x_{4 n-6}=\frac{a^{n-1} f^{n-1}}{c^{n-2} d^{n-1}} \prod_{i=0}^{n-2} \frac{(1+(4 i) c d)}{(1+(4 i+2) a f)}, \quad x_{4 n-5}=\frac{b a^{n-1} f^{n-1}}{c^{n-1} d^{n-1}} \prod_{i=0}^{n-2} \frac{(1+(4 i+1) c d)}{(1+(4 i+3) a f)} \\
& x_{4 n-4}=\frac{a^{n} f^{n-1}}{c^{n-1} d^{n-1}} \prod_{i=0}^{n-2} \frac{(1+(4 i+2) c d)}{(1+(4 i+4) a f)}, \\
& x_{4 n-3}=\frac{a^{n} f^{n}}{e c^{n-1} d^{n-1}(1+a f)} \prod_{i=0}^{n-2} \frac{(1+(4 i+3) c d)}{(1+(4 i+5) a f)}, \\
& y_{4 n-6}=\frac{c^{n-1} d^{n-1}}{a^{n-1} f^{n-2}} \prod_{i=0}^{n-2} \frac{(1+(4 i) a f)}{(1+(4 i+2) c d)}, \\
& y_{4 n-4}=\frac{c^{n-1} d^{n}}{a^{n-1} f^{n-1}} \prod_{i=0}^{n-2} \frac{(1+(4 i+2) a f)}{(1+(4 i+4) c d)}, \\
& y_{4 n-3}=\frac{e c^{n-1} d^{n-1}}{a^{n-1} f^{n-1}} \prod_{i=0}^{n-2} \frac{(1+(4 i+1) a f)}{(1+(4 i+3) c d)} \\
& b a^{n-1} f^{n-1}(1+c d)
\end{aligned}
$$

Now we find from Eq. (1) that

$$
\begin{aligned}
x_{4 n-2}= & \frac{x_{4 n-3} y_{4 n-5}}{y_{4 n-4}\left(1+x_{4 n-3} y_{4 n-5}\right)} \\
= & \left(\frac{a^{n} f^{n}}{e c^{n-1} d^{n-1}(1+a f)} \prod_{i=0}^{n-2} \frac{(1+(4 i+3) c d)}{(1+(4 i+5) a f)}\right)\left(\frac{e c^{n-1} d^{n-1}}{a^{n-1} f^{n-1}} \prod_{i=0}^{n-2} \frac{(1+(4 i+1) a f)}{(1+(4 i+3) c d)}\right) \\
& /\left(\left(\frac{c^{n-1} d^{n}}{a^{n-1} f^{n-1}} \prod_{i=0}^{n-2} \frac{(1+(4 i+2) a f)}{(1+(4 i+4) c d)}\right)\right.
\end{aligned}
$$




$$
\begin{aligned}
& \times\left(1+\left(\frac{a^{n} f^{n}}{e c^{n-1} d^{n-1}(1+a f)} \prod_{i=0}^{n-2} \frac{(1+(4 i+3) c d)}{(1+(4 i+5) a f)}\right)\right. \\
& \left.\left.\times\left(\frac{e c^{n-1} d^{n-1}}{a^{n-1} f^{n-1}} \prod_{i=0}^{n-2} \frac{(1+(4 i+1) a f)}{(1+(4 i+3) c d)}\right)\right)\right) \\
& =\frac{\left(\frac{a f}{(1+a f)} \prod_{i=0}^{n-2} \frac{(1+(4 i+1) a f)}{(1+(4 i+5) a f)}\right)}{\left(\frac{c^{n-1} d^{n}}{a^{n-1} f^{n-1}} \prod_{i=0}^{n-2} \frac{(1+(4 i+2) a f)}{(1+(4 i+4) c d)}\right)\left(1+\frac{a f}{(1+a f)} \prod_{i=0}^{n-2} \frac{(1+(4 i+1) a f)}{(1+(4 i+5) a f)}\right)} \\
& =\frac{\left(\frac{a f}{(1+(4 n-3) a f)}\right)}{\left(\frac{c^{n-1} d^{n}}{a^{n-1} f^{n-1}} \prod_{i=0}^{n-2} \frac{(1+(4 i+2) a f)}{(1+(4 i+4) c d)}\right)\left(1+\frac{a f}{(1+(4 n-3) a f)}\right)} \\
& =\frac{a^{n} f^{n}}{c^{n-1} d^{n}(1+(4 n-2) a f)} \prod_{i=0}^{n-2} \frac{(1+(4 i+4) c d)}{(1+(4 i+2) a f)}=\frac{a^{n} f^{n}}{c^{n-1} d^{n}} \prod_{i=0}^{n-1} \frac{(1+(4 i) c d)}{(1+(4 i+2) a f)}, \\
& y_{4 n-2}=\frac{y_{4 n-3} x_{4 n-5}}{x_{4 n-4}\left(1+y_{4 n-3} x_{4 n-5}\right)} \\
& =\left(\frac{c^{n} d^{n}}{b a^{n-1} f^{n-1}(1+c d)} \prod_{i=0}^{n-2} \frac{(1+(4 i+3) a f)}{(1+(4 i+5) c d)}\right)\left(\frac{b a^{n-1} f^{n-1}}{c^{n-1} d^{n-1}} \prod_{i=0}^{n-2} \frac{(1+(4 i+1) c d)}{(1+(4 i+3) a f)}\right) \\
& /\left(\left(\frac{a^{n} f^{n-1}}{c^{n-1} d^{n-1}} \prod_{i=0}^{n-2} \frac{(1+(4 i+2) c d)}{(1+(4 i+4) a f)}\right)\right. \\
& \times\left(1+\left(\frac{c^{n} d^{n}}{b a^{n-1} f^{n-1}(1+c d)} \prod_{i=0}^{n-2} \frac{(1+(4 i+3) a f)}{(1+(4 i+5) c d)}\right)\right. \\
& \left.\left.\times\left(\frac{b a^{n-1} f^{n-1}}{c^{n-1} d^{n-1}} \prod_{i=0}^{n-2} \frac{(1+(4 i+1) c d)}{(1+(4 i+3) a f)}\right)\right)\right) \\
& =\frac{\frac{c d}{(1+c d)} \prod_{i=0}^{n-2} \frac{(1+(4 i+1) c d)}{(1+(4 i+5) c d)}}{\left(\frac{a^{n} f^{n-1}}{c^{n-1} d^{n-1}} \prod_{i=0}^{n-2} \frac{(1+(4 i+2) c d)}{(1+(4 i+4) a f)}\right)\left(1+\frac{c d}{(1+c d)} \prod_{i=0}^{n-2} \frac{(1+(4 i+1) c d)}{(1+(4 i+5) c d)}\right)} \\
& =\frac{\left(\frac{c d}{(1+(4 n-3) c d)}\right)}{\left(\frac{a^{n} f^{n-1}}{c^{n-1} d^{n-1}} \prod_{i=0}^{n-2} \frac{(1+(4 i+2) c d)}{(1+(4 i+4) a f)}\right)\left(1+\frac{c d}{(1+(4 n-3) c d)}\right)} \\
& =\frac{c^{n} d^{n}}{a^{n} f^{n-1}(1+(4 n-2) c d)} \prod_{i=0}^{n-2} \frac{(1+(4 i+4) a f)}{(1+(4 i+2) c d)}=\frac{c^{n} d^{n}}{a^{n} f^{n-1}} \prod_{i=0}^{n-1} \frac{(1+(4 i) a f)}{(1+(4 i+2) c d)} \text {. }
\end{aligned}
$$

Also, we can prove the other relations. The proof is complete.

The following theorem can be proved similarly.

Theorem 2 Assume that $\left\{x_{n}, y_{n}\right\}$ are solutions of the following system:

$$
x_{n+1}=\frac{x_{n} y_{n-2}}{y_{n-1}\left(1+x_{n} y_{n-2}\right)}, \quad y_{n+1}=\frac{y_{n} x_{n-2}}{x_{n-1}\left(1-y_{n} x_{n-2}\right)} \text {. }
$$

Then for $n=0,1,2, \ldots$,

$$
x_{4 n-2}=\frac{a^{n} f^{n}}{c^{n-1} d^{n}} \prod_{i=0}^{n-1} \frac{(1-(4 i) c d)}{(1+(4 i+2) a f)}, \quad x_{4 n-1}=\frac{b a^{n} f^{n}}{c^{n} d^{n}} \prod_{i=0}^{n-1} \frac{(1-(4 i+1) c d)}{(1+(4 i+3) a f)}
$$




$$
\begin{aligned}
& x_{4 n}=\frac{a^{n+1} f^{n}}{c^{n} d^{n}} \prod_{i=0}^{n-1} \frac{(1-(4 i+2) c d)}{(1+(4 i+4) a f)}, \quad x_{4 n+1}=\frac{a^{n+1} f^{n+1}}{e c^{n} d^{n}(1+a f)} \prod_{i=0}^{n-1} \frac{(1-(4 i+3) c d)}{(1+(4 i+5) a f)}, \\
& y_{4 n-2}=\frac{c^{n} d^{n}}{a^{n} f^{n-1}} \prod_{i=0}^{n-1} \frac{(1+(4 i) a f)}{(1-(4 i+2) c d)}, \\
& y_{4 n}=\frac{c^{n} d^{n+1}}{a^{n} f^{n}} \prod_{i=0}^{n-1} \frac{(1+(4 i+2) a f)}{(1-(4 i+4) c d)},
\end{aligned}
$$

Example 1 For confirming the results of this section, we consider numerical example for the difference system (1) with the initial conditions $x_{-2}=3, x_{-1}=5, x_{0}=-1, y_{-2}=-0.4$, $y_{-1}=0.16$ and $y_{0}=7$. See Figure 1 .

Example 2 We assume that the initial conditions for the difference system (1) are $x_{-2}=$ $-0.2, x_{-1}=0.15, x_{0}=-0.51, y_{-2}=-0.23, y_{-1}=0.16$ and $y_{0}=-0.7$. See Figure 2 .

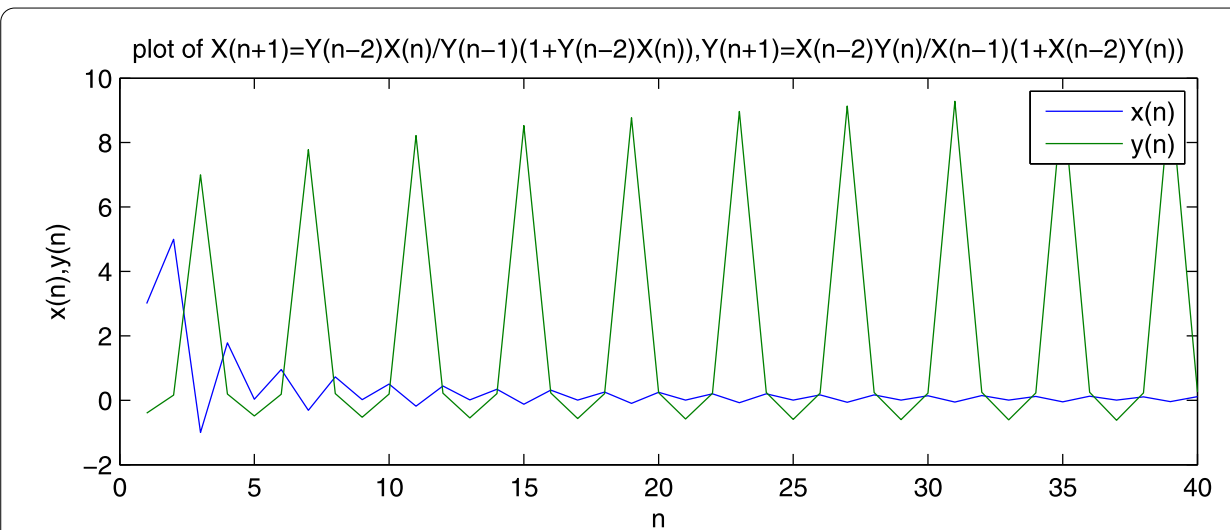

Figure 1 This figure shows the solutions of the system $x_{n+1}=\frac{x_{n} y_{n-2}}{y_{n-1}\left(1+x_{n} y_{n-2}\right)}, y_{n+1}=\frac{y_{n} x_{n-2}}{x_{n-1}\left(1+y_{n} x_{n-2}\right)}$, with the initial conditions $x_{-2}=3, x_{-1}=5, x_{0}=-1, y_{-2}=-0.4, y_{-1}=0.16$ and $y_{0}=7$.

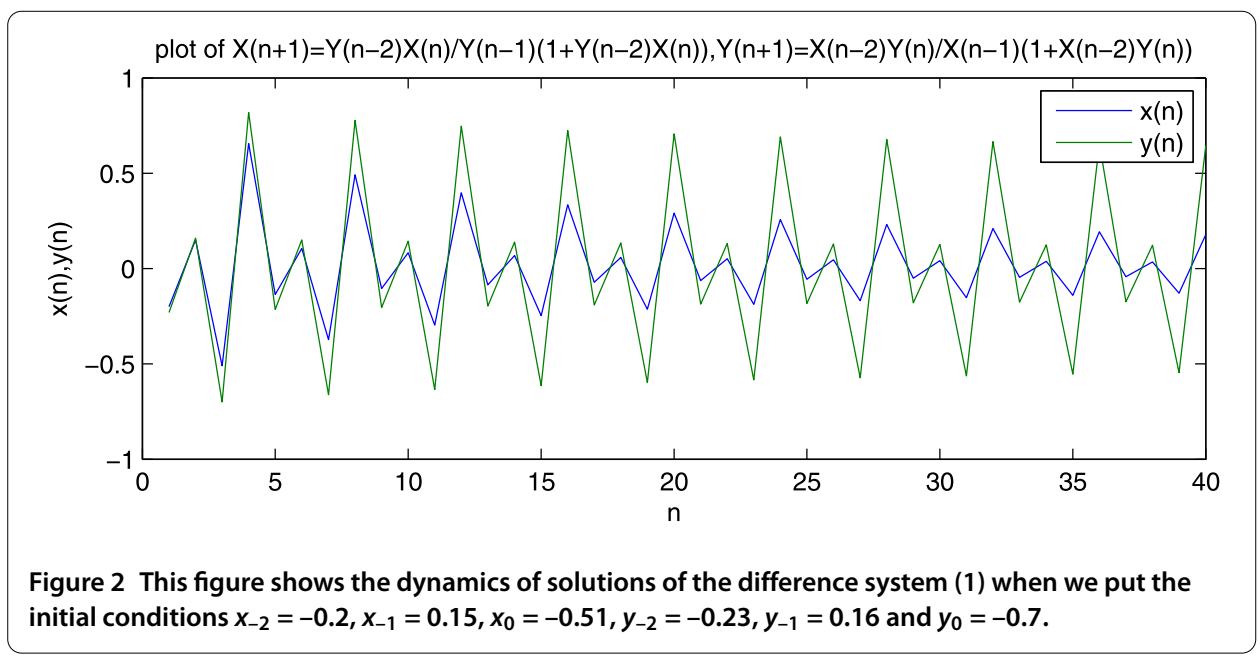


3 On the solution of the system $x_{n+1}=\frac{x_{n} y_{n-2}}{y_{n-1}\left(1+x_{n} y_{n-2}\right)}, y_{n+1}=\frac{y_{n} x_{n-2}}{x_{n-1}\left(-1+y_{n} x_{n-2}\right)}$

In this section, we obtain the form of the solutions of the two difference equations system

$$
x_{n+1}=\frac{x_{n} y_{n-2}}{y_{n-1}\left(1+x_{n} y_{n-2}\right)}, \quad y_{n+1}=\frac{y_{n} x_{n-2}}{x_{n-1}\left(-1+y_{n} x_{n-2}\right)},
$$

where $n \in \mathbb{N}_{0}$ and the initial conditions are arbitrary nonzero real numbers with $x_{-2} y_{0} \neq 1$.

Theorem 3 Let $\left\{x_{n}, y_{n}\right\}_{n=-2}^{+\infty}$ be solutions of system (2). Then $\left\{x_{n}\right\}_{n=-2}^{+\infty}$ and $\left\{y_{n}\right\}_{n=-2}^{+\infty}$ are given by the formula for $n=0,1,2, \ldots$,

$$
\begin{aligned}
& x_{4 n-2}=\frac{a^{n} f^{n}}{c^{n-1} d^{n}} \prod_{i=0}^{n-1} \frac{1}{(1+(4 i+2) a f)}, \quad x_{4 n-1}=\frac{b a^{n} f^{n}}{c^{n} d^{n}} \frac{(-1+c d)^{n}}{\prod_{i=0}^{n-1}(1+(4 i+3) a f)}, \\
& x_{4 n}=\frac{a^{n+1} f^{n}}{c^{n} d^{n}} \prod_{i=0}^{n-1} \frac{1}{(1+(4 i+4) a f)}, \quad x_{4 n+1}=\frac{a^{n+1} f^{n+1}}{e c^{n} d^{n}(1+a f)} \frac{(-1+c d)^{n}}{\prod_{i=0}^{n-1}(1+(4 i+5) a f)}, \\
& y_{4 n-2}=\frac{c^{n} d^{n}}{a^{n} f^{n-1}} \prod_{i=0}^{n-1}(1+(4 i) a f), \\
& y_{4 n}=\frac{c^{n} d^{n+1}}{a^{n} f^{n}} \prod_{i=0}^{n-1}(1+(4 i+2) a f),
\end{aligned}
$$

Proof For $n=0$ the result holds. Now suppose that $n>0$ and that our assumption holds for $n-1$, that is,

$$
\begin{aligned}
& x_{4 n-5}=\frac{b a^{n-1} f^{n-1}}{c^{n-1} d^{n-1}} \frac{(-1+c d)^{n-1}}{\prod_{i=0}^{n-2}(1+(4 i+3) a f)}, \quad x_{4 n-4}=\frac{a^{n} f^{n-1}}{c^{n-1} d^{n-1}} \prod_{i=0}^{n-2} \frac{1}{(1+(4 i+4) a f)}, \\
& x_{4 n-3}=\frac{a^{n} f^{n}}{e c^{n-1} d^{n-1}(1+a f)} \frac{(-1+c d)^{n-1}}{\prod_{i=0}^{n-2}(1+(4 i+5) a f)}, \\
& y_{4 n-5}=\frac{e c^{n-1} d^{n-1}}{a^{n-1} f^{n-1}} \frac{\prod_{i=0}^{n-2}(1+(4 i+1) a f)}{(-1+c d)^{n-1}}, \\
& y_{4 n-4}=\frac{c^{n-1} d^{n}}{a^{n-1} f^{n-1}} \prod_{i=0}^{n-2}(1+(4 i+2) a f), \quad y_{4 n-3}=\frac{c^{n} d^{n}}{b a^{n-1} f^{n-1}} \frac{\prod_{i=0}^{n-2}(1+(4 i+3) a f)}{(-1+c d)^{n}} .
\end{aligned}
$$

Now, we obtain from Eq. (2) that

$$
\begin{aligned}
x_{4 n-2}= & \frac{x_{4 n-3} y_{4 n-5}}{y_{4 n-4}\left(1+x_{4 n-3} y_{4 n-5}\right)} \\
= & \left(\frac{a^{n} f^{n}}{e c^{n-1} d^{n-1}(1+a f)} \frac{(-1+c d)^{n-1}}{\prod_{i=0}^{n-2}(1+(4 i+5) a f)}\right)\left(\frac{e c^{n-1} d^{n-1}}{a^{n-1} f^{n-1}} \frac{\prod_{i=0}^{n-2}(1+(4 i+1) a f)}{(-1+c d)^{n-1}}\right) \\
& /\left(\left(\frac{c^{n-1} d^{n}}{a^{n-1} f^{n-1}} \prod_{i=0}^{n-2}(1+(4 i+2) a f)\right)\right. \\
& \times\left(1+\left(\frac{a^{n} f^{n}}{e c^{n-1} d^{n-1}(1+a f)} \frac{(-1+c d)^{n-1}}{\prod_{i=0}^{n-2}(1+(4 i+5) a f)}\right)\right. \\
& \left.\left.\times\left(\frac{e c^{n-1} d^{n-1}}{a^{n-1} f^{n-1}} \frac{\prod_{i=0}^{n-2}(1+(4 i+1) a f)}{(-1+c d)^{n-1}}\right)\right)\right)
\end{aligned}
$$


Elsayed and El-Metwally Advances in Difference Equations 2013, 2013:161

Page 7 of 14

http://www.advancesindifferenceequations.com/content/2013/1/161

$$
\begin{aligned}
& =\frac{\left(\frac{a f}{(1+(4 n-3) a f)}\right)}{\left(\frac{c^{n-1} d^{n}}{a^{n-1} f^{n-1}} \prod_{i=0}^{n-2}(1+(4 i+2) a f)\right)\left(1+\frac{a f}{(1+(4 n-3) a f)}\right)} \\
& =\frac{a^{n-1} f^{n-1} a f}{\left(c^{n-1} d^{n} \prod_{i=0}^{n-2}(1+(4 i+2) a f)\right)(1+(4 n-3) a f+a f)} \\
& =\frac{a^{n} f^{n}}{\left(c^{n-1} d^{n} \prod_{i=0}^{n-2}(1+(4 i+2) a f)\right)(1+(4 n-2) a f)}=\frac{a^{n} f^{n}}{c^{n-1} d^{n} \prod_{i=0}^{n-1}(1+(4 i+2) a f)} .
\end{aligned}
$$

Also, we can prove the other relations. This completes the proof.

We consider the following systems, and the proof of the theorems is similar to the above theorem, and so it is left to the reader,

$$
\begin{array}{ll}
x_{n+1}=\frac{x_{n} y_{n-2}}{y_{n-1}\left(1+x_{n} y_{n-2}\right)}, & y_{n+1}=\frac{y_{n} x_{n-2}}{x_{n-1}\left(-1-y_{n} x_{n-2}\right)}, \\
x_{n+1}=\frac{x_{n} y_{n-2}}{y_{n-1}\left(1-x_{n} y_{n-2}\right)}, & y_{n+1}=\frac{y_{n} x_{n-2}}{x_{n-1}\left(-1+y_{n} x_{n-2}\right)}, \\
x_{n+1}=\frac{x_{n} y_{n-2}}{y_{n-1}\left(1-x_{n} y_{n-2}\right)}, & y_{n+1}=\frac{y_{n} x_{n-2}}{x_{n-1}\left(-1-y_{n} x_{n-2}\right)} .
\end{array}
$$

The following theorems are devoted to the expressions of the form of the solutions of systems (3), (4), (5) with $x_{-2}=c, x_{-1}=b, x_{0}=a, y_{-2}=f, y_{-1}=e$ and $y_{0}=d$.

Theorem 4 Let $\left\{x_{n}, y_{n}\right\}_{n=-2}^{+\infty}$ be solutions of system (3) and $x_{-2} y_{0} \neq-1$. Then for $n=$ $0,1,2, \ldots$,

$$
\begin{aligned}
& x_{4 n-2}=\frac{a^{n} f^{n}}{c^{n-1} d^{n}} \prod_{i=0}^{n-1} \frac{1}{(1+(4 i+2) a f)}, \quad x_{4 n-1}=\frac{b a^{n} f^{n}}{c^{n} d^{n}} \frac{(-1-c d)^{n}}{\prod_{i=0}^{n-1}(1+(4 i+3) a f)}, \\
& x_{4 n}=\frac{a^{n+1} f^{n}}{c^{n} d^{n}} \prod_{i=0}^{n-1} \frac{1}{(1+(4 i+4) a f)}, \quad x_{4 n+1}=\frac{a^{n+1} f^{n+1}}{e c^{n} d^{n}(1+a f)} \frac{(-1-c d)^{n}}{\prod_{i=0}^{n-1}(1+(4 i+5) a f)}, \\
& y_{4 n-2}=\frac{c^{n} d^{n}}{a^{n} f^{n-1}} \prod_{i=0}^{n-1}(1+(4 i) a f), \\
& y_{4 n}=\frac{c^{n} d^{n+1}}{a^{n} f^{n}} \prod_{i=0}^{n-1}(1+(4 i+2) a f),
\end{aligned}
$$

Theorem 5 Assume that $\left\{x_{n}, y_{n}\right\}$ are solutions of system (4) with $x_{-2} y_{0} \neq 1$. Then for $n=$ $0,1,2, \ldots$,

$$
\begin{aligned}
& x_{4 n-2}=\frac{a^{n} f^{n}}{c^{n-1} d^{n}} \prod_{i=0}^{n-1} \frac{1}{(1-(4 i+2) a f)}, \quad x_{4 n-1}=\frac{b a^{n} f^{n}}{c^{n} d^{n}} \frac{(-1+c d)^{n}}{\prod_{i=0}^{n-1}(1-(4 i+3) a f)}, \\
& x_{4 n}=\frac{a^{n+1} f^{n}}{c^{n} d^{n}} \prod_{i=0}^{n-1} \frac{1}{(1-(4 i+4) a f)}, \quad x_{4 n+1}=\frac{a^{n+1} f^{n+1}}{e c^{n} d^{n}(1+a f)} \frac{(-1+c d)^{n}}{\prod_{i=0}^{n-1}(1-(4 i+5) a f)}, \\
& y_{4 n-2}=\frac{c^{n} d^{n}}{a^{n} f^{n-1}} \prod_{i=0}^{n-1}(1-(4 i) a f),
\end{aligned}
$$




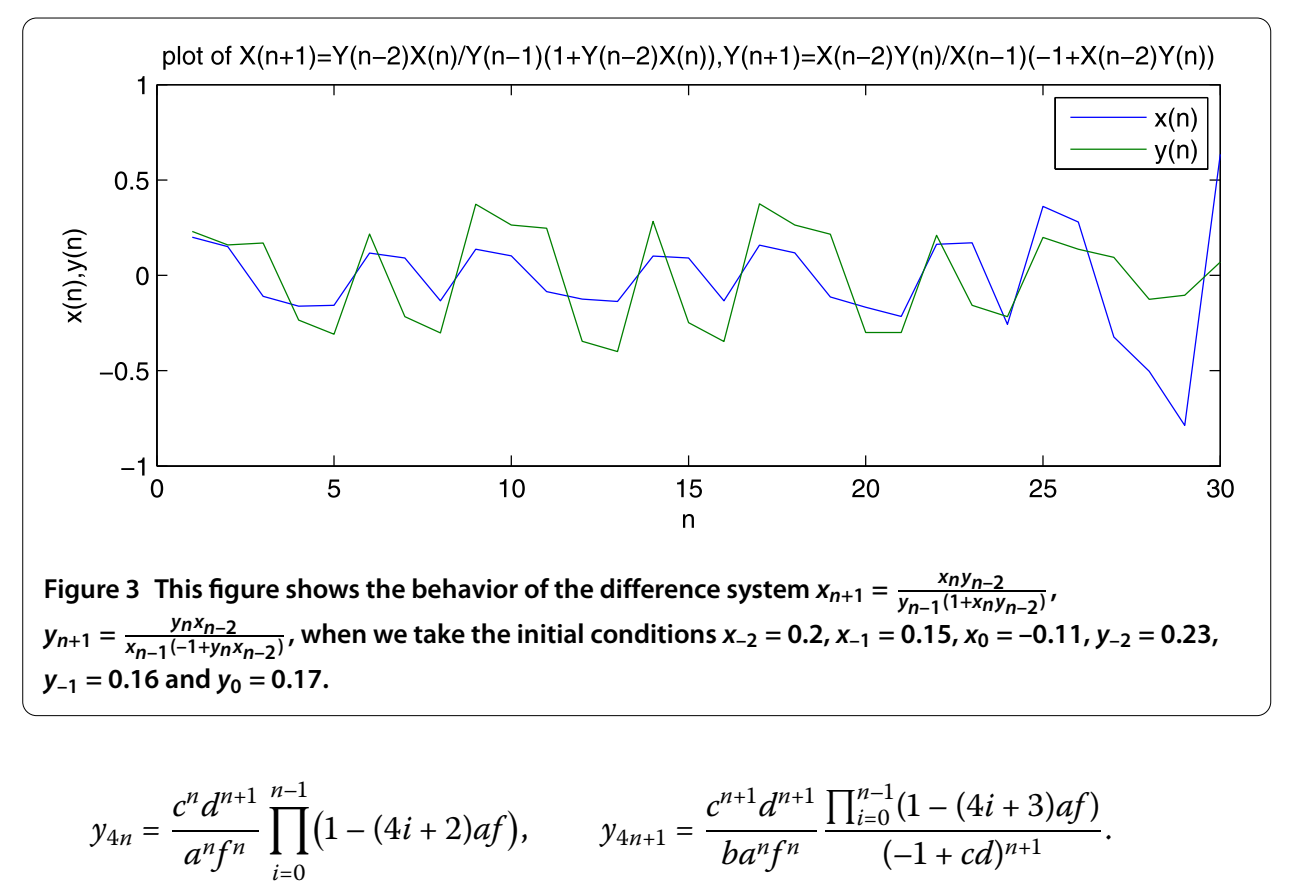

Theorem 6 Suppose that $\left\{x_{n}, y_{n}\right\}$ are solutions of system (5) where $x_{-2} y_{0} \neq-1$. Then for $n=0,1,2, \ldots$,

$$
\begin{array}{lc}
x_{4 n-2}=\frac{a^{n} f^{n}}{c^{n-1} d^{n}} \prod_{i=0}^{n-1} \frac{1}{(1-(4 i+2) a f)}, & x_{4 n-1}=\frac{b a^{n} f^{n}}{c^{n} d^{n}} \frac{(-1-c d)^{n}}{\prod_{i=0}^{n-1}(1-(4 i+3) a f)}, \\
x_{4 n}=\frac{a^{n+1} f^{n}}{c^{n} d^{n}} \prod_{i=0}^{n-1} \frac{1}{(1-(4 i+4) a f)}, & x_{4 n+1}=\frac{a^{n+1} f^{n+1}}{e c^{n} d^{n}(1-a f)} \frac{(-1-c d)^{n}}{\prod_{i=0}^{n-1}(1-(4 i+5) a f)}, \\
y_{4 n-2}=\frac{c^{n} d^{n}}{a^{n} f^{n-1}} \prod_{i=0}^{n-1}(1-(4 i) a f), & y_{4 n-1}=\frac{e c^{n} d^{n}}{a^{n} f^{n}} \frac{\prod_{i=0}^{n-1}(1-(4 i+1) a f)}{(-1-c d)^{n}}, \\
y_{4 n}=\frac{c^{n} d^{n+1}}{a^{n} f^{n}} \prod_{i=0}^{n-1}(1-(4 i+2) a f), & y_{4 n+1}=\frac{c^{n+1} d^{n+1}}{b a^{n} f^{n}} \frac{\prod_{i=0}^{n-1}(1-(4 i+3) a f)}{(-1-c d)^{n+1}} .
\end{array}
$$

Example 3 We consider an interesting numerical example for the difference system (2) with the initial conditions $x_{-2}=0.2, x_{-1}=0.15, x_{0}=-0.11, y_{-2}=0.23, y_{-1}=0.16$ and $y_{0}=$ 0.17. See Figure 3.

Example 4 See Figure 4, where we take system (3) with the initial conditions $x_{-2}=0.12$, $x_{-1}=0.15, x_{0}=0.11, y_{-2}=0.3, y_{-1}=-0.6$ and $y_{0}=0.17$.

4 On the solution of the system $x_{n+1}=\frac{x_{n} y_{n-2}}{y_{n-1}\left(-1+x_{n} y_{n-2}\right)}, y_{n+1}=\frac{y_{n} x_{n-2}}{x_{n-1}\left(-1+y_{n} x_{n-2}\right)}$

In this section, we get the form of the solutions of the difference equations system

$$
x_{n+1}=\frac{x_{n} y_{n-2}}{y_{n-1}\left(-1+x_{n} y_{n-2}\right)}, \quad y_{n+1}=\frac{y_{n} x_{n-2}}{x_{n-1}\left(-1+y_{n} x_{n-2}\right)},
$$

where $n=0,1,2, \ldots$ and the initial conditions $x_{-2}, x_{-1}, x_{0}, y_{-2}, y_{-1}$ and $y_{0}$ are arbitrary nonzero real numbers with $x_{0} y_{-2}, x_{-2} y_{0} \neq 1$. 


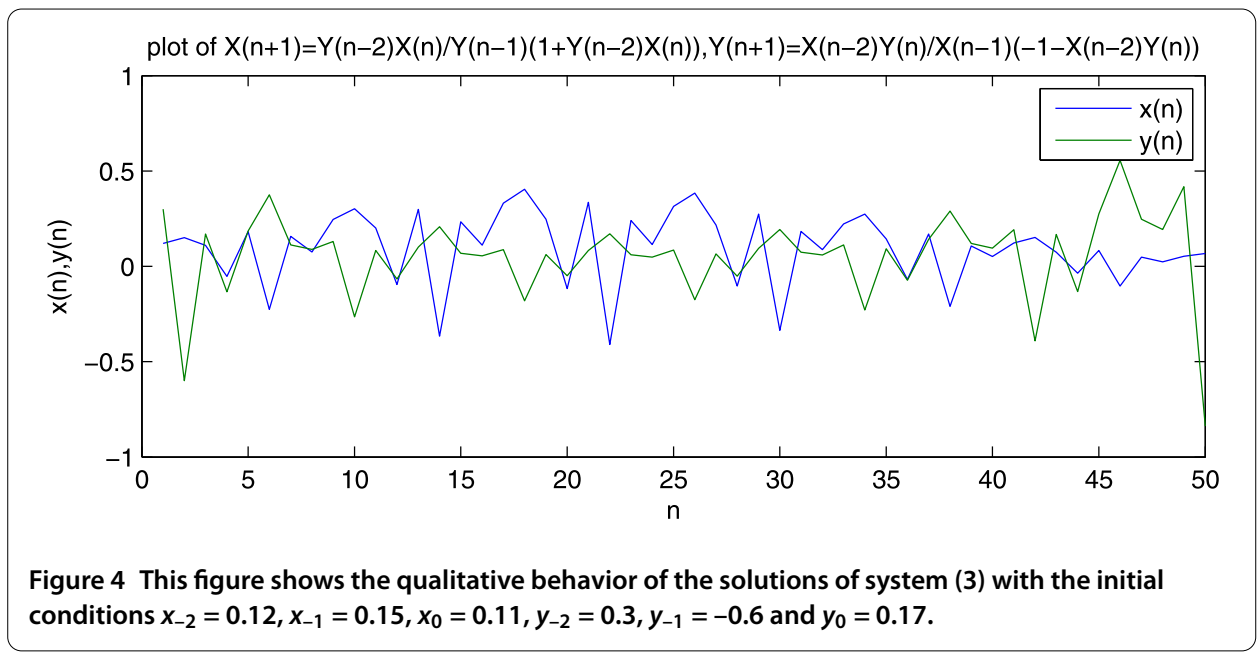

Theorem 7 If $\left\{x_{n}, y_{n}\right\}$ are solutions of difference equation system (6), then for $n=0,1,2, \ldots$,

$$
\begin{aligned}
& x_{4 n-2}=\frac{a^{n} f^{n}}{c^{n-1} d^{n}}, \quad x_{4 n-1}=\frac{b a^{n} f^{n}}{c^{n} d^{n}} \frac{(-1+c d)^{n}}{(-1+a f)^{n}}, \\
& x_{4 n}=\frac{a^{n+1} f^{n}}{c^{n} d^{n}}, \quad x_{4 n+1}=\frac{a^{n+1} f^{n+1}(-1+c d)^{n}}{e c^{n} d^{n}(-1+a f)^{n+1}}, \\
& y_{4 n-2}=\frac{c^{n} d^{n}}{a^{n} f^{n-1}}, \quad y_{4 n-1}=\frac{e c^{n} d^{n}}{a^{n} f^{n}} \frac{(-1+a f)^{n}}{(-1+c d)^{n}}, \\
& y_{4 n}=\frac{c^{n} d^{n+1}}{a^{n} f^{n}}, \quad y_{4 n+1}=\frac{c^{n+1} d^{n+1}(-1+a f)^{n}}{b a^{n} f^{n}(-1+c d)^{n+1}} \text {. }
\end{aligned}
$$

Proof For $n=0$ the result holds. Now, suppose that $n>1$ and that our assumption holds for $n-1$, that is,

$$
\begin{array}{ll}
x_{4 n-6}=\frac{a^{n-1} f^{n-1}}{c^{n-2} d^{n-1}}, & x_{4 n-5}=\frac{b a^{n-1} f^{n-1}(-1+c d)^{n-1}}{c^{n-1} d^{n-1}(-1+a f)^{n-1}}, \\
x_{4 n-4}=\frac{a^{n} f^{n-1}}{c^{n-1} d^{n-1}}, & x_{4 n-3}=\frac{a^{n} f^{n}(-1+c d)^{n-1}}{e c^{n-1} d^{n-1}(-1+a f)^{n}}, \\
y_{4 n-6}=\frac{c^{n-1} d^{n-1}}{a^{n-1} f^{n-2}}, & y_{4 n-5}=\frac{e c^{n-1} d^{n-1}(-1+a f)^{n-1}}{a^{n-1} f^{n-1}(-1+c d)^{n-1}}, \\
y_{4 n-4}=\frac{c^{n-1} d^{n}}{a^{n-1} f^{n-1}}, & y_{4 n-3}=\frac{c^{n} d^{n}(-1+a f)^{n-1}}{b a^{n-1} f^{n-1}(-1+c d)^{n}} .
\end{array}
$$

Now, we conclude from Eq. (6) that

$$
\begin{aligned}
x_{4 n-2} & =\frac{x_{4 n-3} y_{4 n-5}}{y_{4 n-4}\left(-1+x_{4 n-3} y_{4 n-5}\right)}=\frac{\left(\frac{a^{n} f^{n}(-1+c d)^{n-1}}{e c^{n-1} d^{n-1}(-1+a f)^{n}}\right)\left(\frac{e c^{n-1} d^{n-1}(-1+a f)^{n-1}}{a^{n-1} f^{n-1}(-1+c d)^{n-1}}\right)}{\left(\frac{c^{n-1} d^{n}}{a^{n-1} f^{n-1}}\right)\left(-1+\left(\frac{a^{n} f^{n}(-1+c d)^{n-1}}{e c^{n-1} d^{n-1}(-1+a f)^{n}}\right)\left(\frac{e c^{n-1} d^{n-1}(-1+a f)^{n-1}}{a^{n-1} f^{n-1}(-1+c d)^{n-1}}\right)\right)} \\
& =\frac{\left(\frac{a f}{(-1+a f)}\right)}{\left(\frac{c^{n-1} d^{n}}{a^{n-1} f^{n-1}}\right)\left(-1+\frac{a f}{(-1+a f)}\right)}=\frac{a^{n} f^{n}}{c^{n-1} d^{n}},
\end{aligned}
$$




$$
\begin{aligned}
y_{4 n-2} & =\frac{y_{4 n-3} x_{4 n-5}}{x_{4 n-4}\left(-1+y_{4 n-3} x_{4 n-5}\right)}=\frac{\left(\frac{c^{n} d^{n}(-1+a f)^{n-1}}{b a^{n-1} f^{n-1}(-1+c d)^{n}}\right)\left(\frac{b a^{n-1} f^{n-1}(-1+c d)^{n-1}}{c^{n-1} d^{n-1}(-1+a f)^{n-1}}\right)}{\left(\frac{a^{n} f^{n-1}}{c^{n-1} d^{n-1}}\right)\left(-1+\left(\frac{c^{n} d^{n}(-1+a f)^{n-1}}{b a^{n-1} f^{n-1}(-1+c d)^{n}}\right)\left(\frac{b a^{n-1} f^{n-1}(-1+c d)^{n-1}}{c^{n-1} d^{n-1}(-1+a f)^{n-1}}\right)\right)} \\
& =\frac{\left(\frac{c d}{(-1+c d)}\right)}{\left(\frac{a^{n} f^{n-1}}{c^{n-1} d^{n-1}}\right)\left(-1+\left(\frac{c d}{(-1+c d)}\right)\right)}=\frac{c^{n} d^{n}}{a^{n} f^{n-1}} .
\end{aligned}
$$

Also, we can prove the other relations. This completes the proof.

We consider the following system, and the proof of the theorem is similar to the above mentioned theorem and so it is left to the reader,

$$
x_{n+1}=\frac{x_{n} y_{n-2}}{y_{n-1}\left(-1+x_{n} y_{n-2}\right)}, \quad y_{n+1}=\frac{y_{n} x_{n-2}}{x_{n-1}\left(-1-y_{n} x_{n-2}\right)} .
$$

Theorem 8 Let $\left\{x_{n}, y_{n}\right\}_{n=-2}^{+\infty}$ be solutions of system (7) and $x_{0} y_{-2} \neq 1, x_{-2} y_{0} \neq-1$. Then for $n=0,1,2, \ldots$,

$$
\begin{aligned}
& x_{4 n-2}=\frac{a^{n} f^{n}}{c^{n-1} d^{n}}, \quad x_{4 n-1}=\frac{b a^{n} f^{n}}{c^{n} d^{n}} \frac{(-1-c d)^{n}}{(-1+a f)^{n}}, \\
& x_{4 n}=\frac{a^{n+1} f^{n}}{c^{n} d^{n}}, \quad x_{4 n+1}=\frac{a^{n+1} f^{n+1}(-1-c d)^{n}}{e c^{n} d^{n}(-1+a f)^{n+1}}, \\
& y_{4 n-2}=\frac{c^{n} d^{n}}{a^{n} f^{n-1}}, \quad y_{4 n-1}=\frac{e c^{n} d^{n}}{a^{n} f^{n}} \frac{(-1+a f)^{n}}{(-1-c d)^{n}}, \\
& y_{4 n}=\frac{c^{n} d^{n+1}}{a^{n} f^{n}}, \quad y_{4 n+1}=\frac{c^{n+1} d^{n+1}(-1+a f)^{n}}{b a^{n} f^{n}(-1-c d)^{n+1}} \text {. }
\end{aligned}
$$

Lemma 1 The solution of system (6) is unbounded except in the following case.

Theorem 9 System (6) has a periodic solution of period four iff $c d=a f=2$ and it will take the following form: $\left\{x_{n}\right\}=\left\{c, b, a, \frac{a f}{e}, c, b, a, \ldots\right\},\left\{y_{n}\right\}=\left\{f, e, d, \frac{c d}{b}, f, e, d, \ldots\right\}$.

Proof First, suppose that a prime period four solution exists

$$
\left\{x_{n}\right\}=\left\{c, b, a, \frac{a f}{e}, c, b, a, \ldots\right\}, \quad\left\{y_{n}\right\}=\left\{f, e, d, \frac{c d}{b}, f, e, d, \ldots\right\}
$$

of system (6). We see from the form of the solution of system (6) that

$$
\begin{aligned}
& x_{4 n-2}=c=\frac{a^{n} f^{n}}{c^{n-1} d^{n}}, \quad x_{4 n-1}=b=\frac{b a^{n} f^{n}}{c^{n} d^{n}} \frac{(-1+c d)^{n}}{(-1+a f)^{n}}, \\
& x_{4 n}=a=\frac{a^{n+1} f^{n}}{c^{n} d^{n}}, \quad x_{4 n+1}=\frac{a f}{e}=\frac{a^{n+1} f^{n+1}(-1+c d)^{n}}{e c^{n} d^{n}(-1+a f)^{n+1}}, \\
& y_{4 n-2}=f=\frac{c^{n} d^{n}}{a^{n} f^{n-1}}, \quad y_{4 n-1}=e=\frac{e c^{n} d^{n}}{a^{n} f^{n}} \frac{(-1+a f)^{n}}{(-1+c d)^{n}}, \\
& y_{4 n}=d=\frac{c^{n} d^{n+1}}{a^{n} f^{n}}, \quad y_{4 n+1}=\frac{c d}{b}=\frac{c^{n+1} d^{n+1}(-1+a f)^{n}}{b a^{n} f^{n}(-1+c d)^{n+1}} .
\end{aligned}
$$


Then we get $c d=a f=2$. Second, assume that $c d=a f=2$. Then we see from the form of the solution of system (6) that

$$
\begin{array}{lc}
x_{4 n-2}=\frac{a^{n} f^{n}}{c^{n-1} d^{n}}=c, & x_{4 n-1}=\frac{b a^{n} f^{n}}{c^{n} d^{n}} \frac{(-1+c d)^{n}}{(-1+a f)^{n}}=b, \\
x_{4 n}=\frac{a^{n+1} f^{n}}{c^{n} d^{n}}=a, & x_{4 n+1}=\frac{a^{n+1} f^{n+1}(-1+c d)^{n}}{e c^{n} d^{n}(-1+a f)^{n+1}}=\frac{a f}{e}, \\
y_{4 n-2}=\frac{c^{n} d^{n}}{a^{n} f^{n-1}}=f, & y_{4 n-1}=\frac{e c^{n} d^{n}}{a^{n} f^{n}} \frac{(-1+a f)^{n}}{(-1+c d)^{n}}=e, \\
y_{4 n}=\frac{c^{n} d^{n+1}}{a^{n} f^{n}}=c, & y_{4 n+1}=\frac{c^{n+1} d^{n+1}(-1+a f)^{n}}{b a^{n} f^{n}(-1+c d)^{n+1}}=\frac{c d}{b} .
\end{array}
$$

Thus, we have a periodic solution of period four and the proof is complete.

Lemma 2 The solution of system (7) is unbounded except in the following case.

Theorem 10 System (7) has a periodic solution of period eight iff $c d=-2$, af $=2$ and it will take the following form: $\left\{x_{n}\right\}=\left\{c, b, a, \frac{a f}{e},-c,-b,-a,-\frac{a f}{e}, c, b, a, \ldots\right\},\left\{y_{n}\right\}=$ $\left\{f, e, d, \frac{c d}{b},-f,-e,-d,-\frac{c d}{b}, f, e, d, \ldots\right\}$.

Example 5 We consider a numerical example for the difference system (6) when we put the initial conditions $x_{-2}=0.12, x_{-1}=0.5, x_{0}=0.31, y_{-2}=0.23, y_{-1}=-0.6$ and $y_{0}=0.7$. See Figure 5.

Example 6 Figure 6 shows the behavior of the solution of the difference system (6) with the initial conditions $x_{-2}=4, x_{-1}=-7, x_{0}=-0.5, y_{-2}=-4, y_{-1}=6$ and $y_{0}=0.5$.

Example 7 We consider a numerical example for the difference system (7) when we put the initial conditions $x_{-2}=0.32, x_{-1}=0.25, x_{0}=-0.31, y_{-2}=0.23, y_{-1}=0.26$ and $y_{0}=0.17$. See Figure 7.

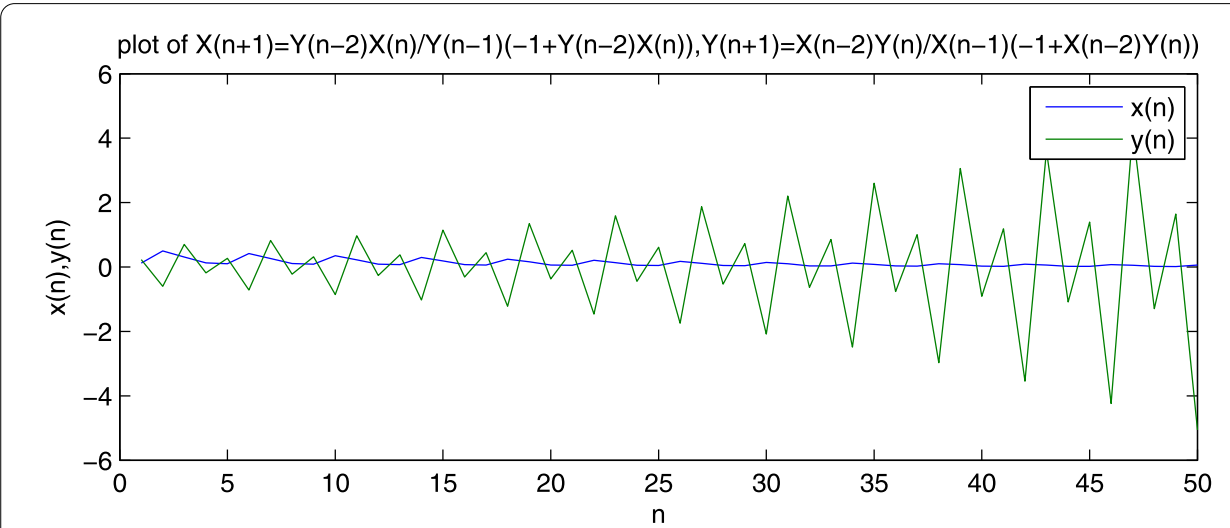

Figure 5 This figure shows the solution of the system of difference equations $x_{n+1}=\frac{x_{n} y_{n-2}}{y_{n-1}\left(-1+x_{n} y_{n-2}\right)}$, $y_{n+1}=\frac{y_{n} x_{n-2}}{x_{n-1}\left(-1+y_{n} x_{n-2}\right)}$, when we put the initial conditions $x_{-2}=0.12, x_{-1}=0.5, x_{0}=0.31, y_{-2}=0.23$, $y_{-1}=-0.6$ and $y_{0}=0.7$. 

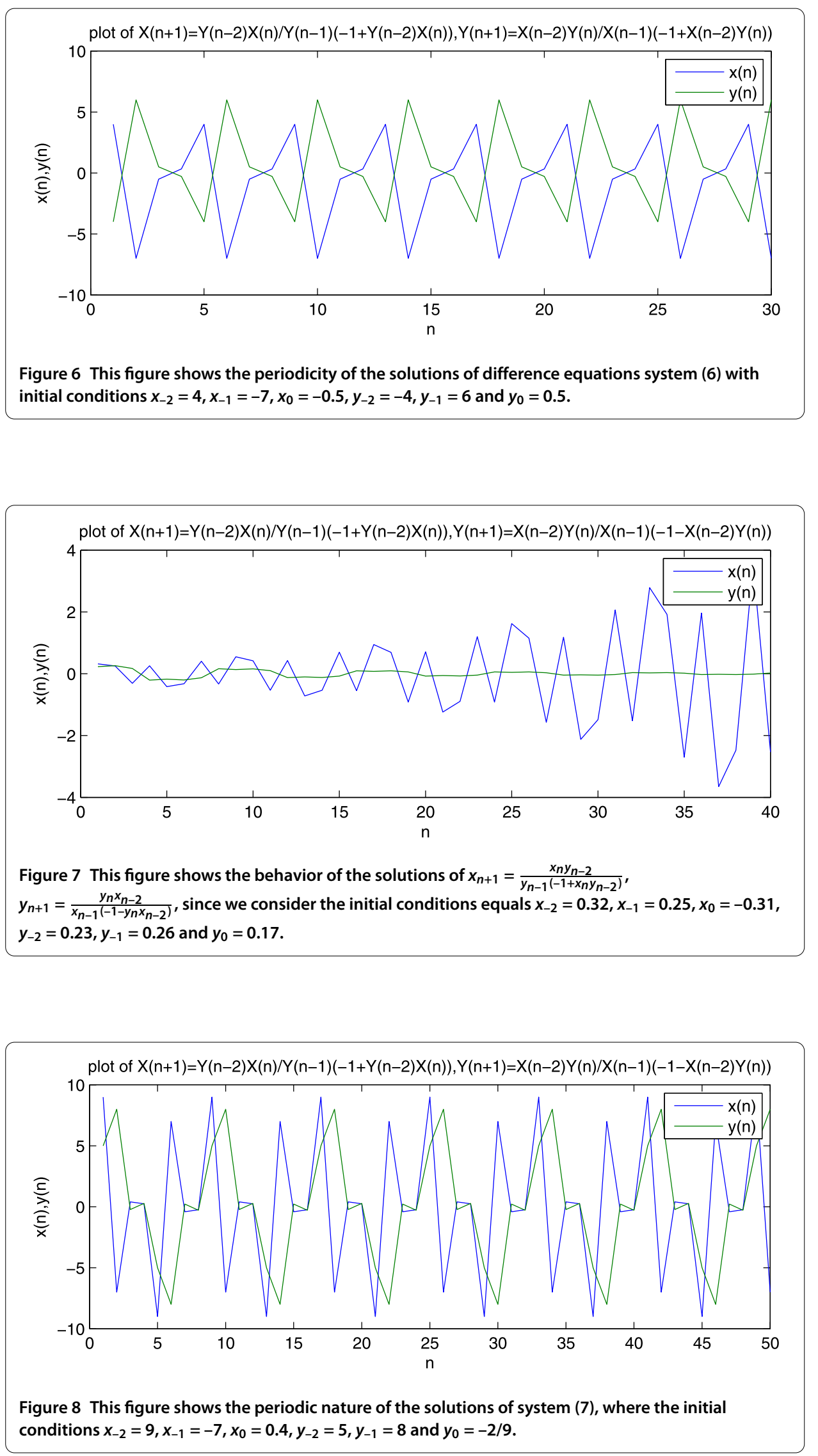
Example 8 Figure 8 shows the periodicity of the solution of the difference system (7) with the initial conditions $x_{-2}=9, x_{-1}=-7, x_{0}=0.4, y_{-2}=5, y_{-1}=8$ and $y_{0}=-2 / 9$.

\section{Competing interests}

The authors declare that they have no competing interests.

\section{Authors' contributions}

The authors declare that the study was realized in collaboration with the same responsibility. Both authors read and approved the final manuscript.

\section{Author details}

${ }^{1}$ King Abdulaziz University, Faculty of Science, Mathematics Department, P.O. Box 80203, Jeddah 21589, Saudi Arabia. 2Department of Mathematics, Rabigh College of Science and Art, King Abdulaziz University, P.O. Box 344, Rabigh 21911, Saudi Arabia. ${ }^{3}$ Department of Mathematics, Faculty of Science, Mansoura University, Mansoura 35516, Egypt.

\section{Acknowledgements}

This work was funded by the Deanship of Scientific Research (DSR), King Abdulaziz University, Jeddah, under grant No. (130-028-D1433). The authors, therefore, acknowledge with thanks DSR technical and financial support.

Received: 11 December 2012 Accepted: 23 May 2013 Published: 7 June 2013

\section{References}

1. Cull, P, Flahive, M, Robson, R: Difference Equations: From Rabbits to Chaos. Undergraduate Texts in Mathematics. Springer, New York (2005)

2. Elaydi, S: An Introduction to Difference Equations, 3rd edn. Undergraduate Texts in Mathematics. Springer, New York (2005)

3. Beverton, RJH, Holt, SJ: On the Dynamics of Exploited Fish Populations. Fishery Investigations Series II, vol. 19. Blackburn Press, Caldwell (2004)

4. Ahlbrandt, CD, Peterson, AC: Discrete Hamiltonian Systems: Difference Equations, Continued Fractions, and Riccati Equations. Kluwer Texts in the Mathematical Sciences, vol. 16. Kluwer Academic, Dordrecht (1996)

5. Kocic, VL, Ladas, G: Global Behavior of Nonlinear Difference Equations of Higher Order with Applications. Kluwer Academic, Dordrecht (1993)

6. Kulenovic, MRS, Ladas, G: Dynamics of Second Order Rational Difference Equations with Open Problems and Conjectures. Chapman \& Hall/CRC Press, Boca Raton (2001)

7. Camouzis, E, Kulenovic, MRS, Ladas, G, Merino, O: Rational systems in the plane. J. Differ. Equ. Appl. 15(3), 303-323 (2009)

8. Cinar, C, Yalçinkaya, I, Karatas, R: On the positive solutions of the difference equation system $x_{n+1}=m / y_{n}$, $y_{n+1}=p y_{n} / x_{n-1} y_{n-1}$. J. Inst. Math. Comput. Sci. 18, 135-136 (2005)

9. Clark, D, Kulenovic, MRS: A coupled system of rational difference equations. Comput. Math. Appl. 43, 849-867 (2002)

10. Kurbanli, AS, Cinar, C, Yalçınkaya, I: On the behavior of positive solutions of the system of rational difference equations. Math. Comput. Model. 53, 1261-1267 (2011)

11. Touafek, N, Elsayed, EM: On the solutions of systems of rational difference equations. Math. Comput. Model. 55 , 1987-1997 (2012)

12. Yalcinkaya, I, Cinar, C, Atalay, M: On the solutions of systems of difference equations. Adv. Differ. Equ. 2008, Article ID $143943(2008)$

13. Agarwal, RP: Difference Equations and Inequalities, 1st edn. Dekker, New York (1992) (2nd edn. (2000))

14. Agarwal, RP, Elsayed, EM: On the solution of fourth-order rational recursive sequence. Adv. Stud. Contemp. Math. 20(4), 525-545 (2010)

15. Battaloglu, N, Cinar, C, Yalçınkaya, I: The dynamics of the difference equation. Ars Comb. 97, 281-288 (2010)

16. Din, Q, Qureshi, MN, Qadeer Khan, A: Dynamics of a fourth-order system of rational difference equations. Adv. Differ. Equ. 2012, 215 (2012)

17. Elabbasy, EM, El-Metwally, H, Elsayed, EM: On the solutions of a class of difference equations systems. Demonstr. Math. 41(1), 109-122 (2008)

18. Elabbasy, EM, El-Metwally, H, Elsayed, EM: Global behavior of the solutions of difference equation. Adv. Differ. Equ. 2011, 28 (2011)

19. Elsayed, EM: Solution and attractivity for a rational recursive sequence. Discrete Dyn. Nat. Soc. 2011, Article ID 982309 (2011)

20. Elsayed, EM: On the solution of some difference equations. Eur. J. Pure Appl. Math. 4(3), 287-303 (2011)

21. Elsayed, EM: Behavior and expression of the solutions of some rational difference equations. J. Comput. Anal. Appl. 15(1), 73-81 (2013)

22. Elsayed, EM: Solutions of rational difference system of order two. Math. Comput. Model. 55, 378-384 (2012)

23. Elsayed, EM, El-Dessoky, MM: Dynamics and behavior of a higher order rational recursive sequence. Adv. Differ. Equ. 2012, 69 (2012)

24. Elsayed, EM, El-Dessoky, M, Alotaibi, A: On the solutions of a general system of difference equations. Discrete Dyn. Nat. Soc. 2012, Article ID 892571 (2012)

25. Keying, L, Zhongjian, Z, Xiaorui, L, Peng, L: More on three-dimensional systems of rational difference equations. Discrete Dyn. Nat. Soc. 2011, Article ID 178483 (2011)

26. Kurbanli, AS: On the behavior of solutions of the system of rational difference equations. Adv. Differ. Equ. 2011,40 (2011) 
27. Mansour, M, El-Dessoky, MM, Elsayed, EM: The form of the solutions and periodicity of some systems of difference equations. Discrete Dyn. Nat. Soc. 2012, Article ID 406821 (2012)

28. Papaschinopoulos, G, Radin, MA, Schinas, CJ: On the system of two difference equations of exponential form: $x_{n+1}=a+b x_{n-1} e^{-y_{n}}, y_{n+1}=c+d y_{n-1} e^{-x_{n}}$. Math. Comput. Model. 54(11-12), 2969-2977 (2011)

29. Papaschinopoulos, G, Schinas, CJ: On the dynamics of two exponential type systems of difference equations Comput. Math. Appl. 64(7), 2326-2334 (2012)

30. Touafek, N, Elsayed, EM: On the periodicity of some systems of nonlinear difference equations. Bull. Math. Soc. Sci. Math. Roum. 55(103)(2), 217-224 (2012)

31. Yalçınkaya, I: On the global asymptotic behavior of a system of two nonlinear difference equations. Ars Comb. 95 151-159 (2010)

32. Yang, $X, L i u, Y, B a i, S$ : On the system of high order rational difference equations $x_{n}=\frac{a}{y_{n-p}}, y_{n}=\frac{b y_{n-p}}{x_{n-q} y_{n-q}}$. Appl. Math. Comput. 171(2), 853-856 (2005)

33. Wang, C, Wang, S, Wang, W: Global asymptotic stability of equilibrium point for a family of rational difference equations. Appl. Math. Lett. 24(5), 714-718 (2011)

34. Wang, C, Shi, Q, Wang, S: Asymptotic behavior of equilibrium point for a family of rational difference equation. Adv. Differ. Equ. 2010, Article ID 505906 (2010)

35. Wang, C, Gong, F, Wang, S, Li, L, Shi, Q: Asymptotic behavior of equilibrium point for a class of nonlinear difference equation. Adv. Differ. Equ. 2009, Article ID 214309 (2009)

36. Wang, C, Wang, S, Wang, Z, Gong, F, Wang, R: Asymptotic stability for a class of nonlinear difference equation. Discrete Dyn. Nat. Soc. 2010, Article ID 791610 (2010)

37. Zayed, EME, El-Moneam, MA: On the rational recursive sequence $x_{n+1}=A x_{n}+\left(\beta x_{n}+\gamma x_{n-k}\right) /\left(C x_{n}+D x_{n-k}\right)$. Commun Appl. Nonlinear Anal. 16, 91-106 (2009)

38. Zhang, Q, Yang, L, Liu, J: Dynamics of a system of rational third order difference equation. Adv. Differ. Equ. 2012, 136 (2012)

39. Zkan, O, Kurbanli, AS: On a system of difference equation. Discrete Dyn. Nat. Soc. 2013, Article ID 970316 (2013)

doi:10.1186/1687-1847-2013-16

Cite this article as: Elsayed and El-Metwally: On the solutions of some nonlinear systems of difference equations. Advances in Difference Equations 2013 2013:161

\section{Submit your manuscript to a SpringerOpen ${ }^{\odot}$ journal and benefit from:}

- Convenient online submission

- Rigorous peer review

- Immediate publication on acceptance

- Open access: articles freely available online

High visibility within the field

- Retaining the copyright to your article 